\title{
Effect of Endoscopic Sinus Surgery on Clinical Outcomes in Cystic Fibrosis
}

\author{
Anali Dadgostar ${ }^{1}$, Sepehr Nassiri ${ }^{1}$, Bradley Quon ${ }^{2}$, Jamil Manji ${ }^{3}$, Salahuddin Alsalihi ${ }^{1}$, and \\ Amin Javer ${ }^{1}$ \\ ${ }^{1}$ St Pauls Sinus Centre \\ ${ }^{2}$ The University of British Columbia Centre for Heart Lung Innovation \\ ${ }^{3}$ The University of Melbourne Faculty of Medicine Dentistry and Health Sciences
}

May 18, 2020

\begin{abstract}
Objectives: Chronic rhinosinusitis (CRS) is prevalent in the Cystic Fibrosis (CF) population. CRS exacerbations in CF are thought to contribute to pulmonary exacerbations. Literature regarding the impact of endoscopic sinus surgery (ESS) is inconclusive. This study examines rates of lung function decline and pulmonary exacerbation in CF patients who have undergone ESS. Design: Retrospective review of medical records. Setting: Academic Hospital. Participants: 40 adult CF patients. Main outcome measures: Rate of lung function decline (Forced Expiratory Volume1(FEV1) \% predicted), number of pulmonary exacerbations (IV/oral antibiotic therapy +/- hospital admission) and total number days hospitalized 2 years post-operatively was collected. CRS patients undergoing ESS were matched to those without ESS by gender, age, and F508del genotype. Results: Forty patients (mean age 37.4, 60\% male) were reviewed. No significant difference was found between the surgical group and controls in baseline $\operatorname{FEV1}(72.5 \%$ vs. $72.7 \%$, p=0.98), 2-year pre-operative number of pulmonary exacerbations (3.05 vs. 1.65 , $\mathrm{p}=0.10)$, or Lund-Mackay scores (12.25 vs. $11.55, \mathrm{p}=0.71)$. No significant difference was found in 1 -year $(70.5 \%$ vs. $72.8 \%$, $\mathrm{p}=0.84)$ or 2-year $(70.4 \%$ vs. $72.6 \% \mathrm{p}=0.80)$ post-operative FEV1 and 2-year post-operative pulmonary exacerbations (1.7 vs. 1.45, $\mathrm{p}=0.87)$. A significant increase was identified in total number days hospitalized post-operatively $(4.85, \mathrm{p}=0.02)$. In the surgical group, no significant difference was identified between preoperative and postoperative FEV1, $1-$ year $(-2.51 \%$, $\mathrm{p}=0.32)$ and 2 -years after $\operatorname{ESS}(-3.10 \%, \mathrm{p}=0.51)$, postoperative rate of pulmonary exacerbations $(-1.28, \mathrm{p}=0.11)$, or in total number days hospitalized $(3.74, \mathrm{p}=0.14)$. Conclusions: In this study, ESS does not appear to significantly improve FEV1 or decrease the number of pulmonary exacerbations post-operatively.
\end{abstract}

\section{ABSTRACT}

Objectives: Chronic rhinosinusitis (CRS) is prevalent in the Cystic Fibrosis (CF) population. CRS exacerbations in $\mathrm{CF}$ are thought to contribute to pulmonary exacerbations. Literature regarding the impact of endoscopic sinus surgery (ESS) is inconclusive. This study examines rates of lung function decline and pulmonary exacerbation in CF patients who have undergone ESS.

Design : Retrospective review of medical records.

Setting: Academic Hospital.

Participants: 40 adult CF patients.

Main outcome measures: Rate of lung function decline (Forced Expiratory Volume1(FEV1) \% predicted), pulmonary exacerbations (IV/oral antibiotic therapy +/- hospital admission) and total days hospitalized 2 years post-operatively was collected. CRS patients undergoing ESS were matched to those without ESS by gender, age, and F508del genotype. 
Results: Forty patients (mean age 37.4, 60\% male) were reviewed. No significant difference was found between the surgical group and controls in baseline FEV1(72.5\% vs. $72.7 \%, \mathrm{p}=0.98), 2$-year pre-operative pulmonary exacerbations ( 3.05 vs. $1.65, \mathrm{p}=0.10)$, or Lund-Mackay scores $(12.25$ vs. $11.55, \mathrm{p}=0.71)$. No significant difference was found in 1 -year $(70.5 \%$ vs. $72.8 \%, \mathrm{p}=0.84)$ or 2 -year $(70.4 \%$ vs. $72.6 \% \mathrm{p}=0.80)$ post-operative FEV1 and 2-year post-operative pulmonary exacerbations (1.7 vs. $1.45, \mathrm{p}=0.87)$. A significant increase was identified in total number days hospitalized post-operatively $(4.85, \mathrm{p}=0.02)$. In the surgical group, no significant difference was identified between preoperative and postoperative FEV1, 1 -year (-2.51\%, $\mathrm{p}=0.32)$ and 2-years after $\operatorname{ESS}(-3.10 \%, \mathrm{p}=0.51)$, postoperative pulmonary exacerbations $(-1.28, \mathrm{p}=0.11)$, or in days hospitalized $(3.74, \mathrm{p}=0.14)$.

Conclusions: In this study, ESS does not appear to significantly improve FEV1 or decrease the number of pulmonary exacerbations post-operatively.

Keywords: Cystic Fibrosis, Sinus Surgery, Outcomes, Lung Function, Sinusitis, Forced Expiratory Volume, Nasal polyposis

\section{INTRODUCTION}

Cystic fibrosis (CF) is a genetic disorder involving the upper and lower airways and digestive system, affecting 30,000 children and adults in the United States, 4,000 in Canada, and 70,000 worldwide $[1,2]$. CF is an autosomal recessive disease based on a mutation in the CF transmembrane conductance regulator (CFTR) gene. The CFTR gene is encoded on the q31 region of the long arm of chromosome 7 [3]. The CFTR protein regulates the components of sweat, digestive fluid and mucus by controlling the transport of chloride ions across epithelial membranes. In CF, lack of functional CFTR impairs chloride and bicarbonate transport across the apical epithelium producing thickened secretions, dysfunctional mucociliary clearance, and chronic airway bacterial infection [4]. Imbalance of electrolyte transport from CFTR dysfunction reduces airway surface liquid depth and increases the viscosity of mucins in the airway 30-60 times higher than seen in patients without $\mathrm{CF}$ [5]. The mucostasis results in progressive cycles of infection and inflammation in the respiratory tract, culminating in respiratory failure. Although advances in the clinical care of the CF patient have increased the average lifespan, the most common cause of mortality continues to be airway disease in the form of progressive bronchiectasis and ultimately respiratory failure [6].

Sinonasal disease in the form of chronic rhinosinusitis, with or without nasal polyposis, is prevalent in the CF population. Radiologic findings of paranasal sinus opacification on computed tomography can be found in almost $100 \%$ of patients with $\mathrm{CF}$, with a reported incidence of nasal polyposis between $33 \%$ and $57 \%$ [7-9]. Exacerbations of rhinosinusitis in $\mathrm{CF}$ are thought to contribute to pulmonary exacerbations that may suggest transmission of bacterial infection from the upper to the lower airway. Support for this association includes studies establishing the presence of similar bacteria in pulmonary and sinonasal cultures [10-12]. Colonization of both the upper and lower airways with Pseudomonas aeruginosa is a well-known phenomenon in patients with cystic fibrosis (CF) [13]. These findings indicate the sinuses act as a bacterial reservoir for transmitting disease to the lower airways, making aggressive treatment and ongoing management of sinonasal disease a priority for improving pulmonary outcomes [14].

Current literature regarding the effect of sinus surgery and management of chronic rhinosinusitis on pulmonary and clinical outcomes in the cystic fibrosis population is conflicting. This study will review a 10-year experience at '[removed for blind peer review]'.comparing outcomes in cystic fibrosis patients. Specifically, this study examines the rates of lung function decline (i.e. FEV $1 \%$ predicted) and pulmonary exacerbation rates (i.e. need for IV antibiotics/hospitalization) in CF patients who have undergone endoscopic sinus surgery as compared to a group of CF patients with CT or endoscopic findings of chronic rhinosinusitis but without endoscopic sinus surgery.

\section{MATERIALS AND METHODS}

\section{Study Design}

A retrospective review of medical records from '[removed for blind peer review]'.between 2005 and 2015 was 
performed. Data on FEV-1 \% predicted and number of pulmonary exacerbations were collected as available in the patient chart. Lung function decline (FEV $1 \%$ predicted, in the first 2 years post-operatively) was examined. Pulmonary exacerbation rate (ie. number of pulmonary exacerbations) was defined by treatment with IV or oral antibiotic therapy +/- hospital admission for pulmonary exacerbation. Total number of days hospitalized 2 years pre-operatively and 2 years post-operatively for acute infections were identified and recorded. Lund-Mackay CT scores were determined for all patients.

\section{Study Population}

All cystic fibrosis patients over the age of 19 evaluated at '[removed for blind peer review]'.were included in the review. Patients were included if they held a diagnosis of with CF (positive sweat chloride test +/genetic phenotype testing) and diagnosis of chronic rhinosinusitis (CRS) (with/without polyposis) based on the Canadian Rhinosinusitis Guidelines (may include radiologic or endoscopic findings of CRS) [15]. Patients were excluded if they had prior lung transplantation or were diagnosed with sinonasal tumors. 100 patients were reviewed, 40 were included in the study. A significant number of patients had missing data points including date of surgery or FEV1 levels.

All surgical patients underwent computer-assisted endoscopic sinus surgery, consisting of bilateral maxillary antrostomy, anterior and posterior ethmoidectomy, sphenoidotomy, and frontal sinusotomy (when present). None of the patients were treated with topical inhaled antibiotics.

Patients in the surgical group $(\mathrm{n}=20)$ were matched 1:1 to non-surgical counterparts based on the covariates of gender, age, and mutation (F508del homozygous vs heterozygous). Although initially approximately 100 patients were reviewed, Once matched, FEV-1 \% predicted 1-year pre-surgical date, FEV-1\% predicted 1year post-surgical date, and FEV-1\% predicted 2-years post-surgical date were identified. The total number of pulmonary exacerbations and the total number of days hospitalized 2 years pre and 2 years post-surgical date were identified and recorded.

\section{Statistical Analyses}

Statistical software (STATA), version 14.2 was used for statistical analysis. Linear regression was used to analyze the continuous FEV1 \% predicted variable, number of pulmonary exacerbations, and total number of days hospitalized in the surgical versus non-surgical groups, adjusting for correlational data analysis and effective size measurement. Standard errors were inflated to reflect the degree of correlation. Unadjusted and adjusted analysis was calculated.

\section{RESULTS}

Forty patients with CF (mean age 37.4 years, 60\% male) were reviewed. Non-surgical patients appear to have higher rates of Pseudomonas, whereas surgical patients have higher rates of MRSA airway colonization (Table 1). Pre-operative and post-operative respiratory cultures (sputum, bronchioalveolar lavage, or sinus culture) were obtained for 11 out of 20 surgical patients. All 11 patients had the same bacterial culture pre-operatively and post-operatively. All 3 of the 11 surgical patients with no growth pre-operatively did not have bacterial growth post-operatively (Table 2).

No significant difference was found between the surgical group and matched non-surgical controls in baseline FEV1 (72.5\% vs. $72.7 \%, \mathrm{p}=0.98)$. The 2 -year pre-operative number of pulmonary exacerbations did not show any statistically significant difference $(3.05$ vs. $1.65, \mathrm{p}=0.10)$ between the two groups. No significant difference was found between the surgical group and matched non-surgical controls in pre-operative LundMackay scores (12.25 vs. $11.55, \mathrm{p}=0.71$ ). The 2-year pre-operative total number of days hospitalized did not show any statistically significant difference ( 4.5 vs. $3.41, \mathrm{p}=0.60)$ between the two groups. (Table 3 ).

Post-operatively, no significant difference between the surgical group and matched non-surgical controls was found in the 1-year post-operative FEV1 (70.5\% vs. 72.8\%, $\mathrm{p}=0.84$ ), 2-year post-operative FEV1 (70.4\% vs. $72.6 \% \mathrm{p}=0.80)$ and 2-year post-operative number of pulmonary exacerbations (1.7 vs. $1.45, \mathrm{p}=0.87)$. There 
was a significant increase in the 2 year post-operative total number of days hospitalized in the surgical group (6.2 vs. $1.6, \mathrm{p}=0.03)$ (Table 4$)$.

In the surgical group alone, although the trend appeared to show a decrease in FEV1 post-operatively, no significant difference was identified in the change in FEV1 (preoperative to post-operative) at 1 -year postoperatively $(-2.51 \%, \mathrm{p}=0.32)$ and 2 -years post-operatively $(-3.10 \%, \mathrm{p}=0.51)$. Although the rate of pulmonary exacerbations post-operatively appears to decrease, this value is not significant $(-1.28, \mathrm{p}=0.11)$. The total number of days hospitalized 2 years post-operatively did show a trend towards an increase within the surgical group itself, however, this value was not statistically significant $(3.74, \mathrm{p}=0.14)$. (Table 5$)$.

\section{DISCUSSION}

\section{Synopsis of Key Findings}

This study examines the rates of lung function decline and pulmonary exacerbations in a cohort of CF patients who have undergone endoscopic sinus surgery as compared to a group of CF patients with CT or endoscopic findings of chronic rhinosinusitis but without endoscopic sinus surgery. Our study demonstrates 4 main findings: 1) non-surgical patients appear to have higher rates of pseudomonas airway colonization while surgical patients appear to have higher rates of MRSA colonization; surgical intervention does not appear to change bacterial colonization, 2) sinus surgery does not appear to significantly improve FEV-1\% predicted post-operatively, 3) sinus surgery does not appear to significantly decrease number of pulmonary exacerbations post-operatively, and 4) sinus surgery appears to increase the total number of days hospitalized post-operatively.

\section{Comparisons with Other Studies}

Current literature regarding the benefit of endoscopic sinus surgery in the CF population is conflicting. A few studies suggest that sinus surgery and management of CRS can lead to improved pulmonary and clinical outcomes in the CF population, while many others have not shown much benefit. Shatz [16] examined a cohort of 15 pediatric patients with $\mathrm{CF}$ and found improvements in pulmonary function test results, fewer courses of IV antibiotics, and a significant decrease in number of hospital admissions (all p < 0.006) after sinus surgery. This cohort of patients underwent aggressive combined surgery (open and endoscopic) and had previously undergone least 3-revision surgeries.

Liang et al [17] performed a systematic review of surgical management of chronic rhinosinusitis in cystic fibrosis encompassing the last 25 years with 24 articles meeting their inclusion criteria. The outcomes examined included sinonasal symptoms, endoscopic findings, pulmonary function testing, recurrence or revision surgery, hospitalization, need for antibiotic therapy, radiographic findings, and pulmonary exacerbations. Most studies in their review found improvement in symptom measures and endoscopic findings with no improvement in lower airway function after surgical therapy. The postoperative measures of the other outcomes were inconclusive or inconsistent.

In a systematic review examining the role of endoscopic sinus surgery on pulmonary function in patients with cystic fibrosis, nineteen studies involving 586 patients were analyzed [18]. The authors found consistent evidence of improved sinonasal symptoms. Overall, pulmonary function tests were not improved by ESS in six cohort trials, with one small study finding significant improvement. No significant difference in FEV1 scores was confirmed by meta-analysis and no consistent improvement in pulmonary function testing. Conflicting results with regards to endoscopy scores, days spent in hospital, and courses of intravenous antibiotics was identified.

Henriquez et al [19] examined the effect of endoscopic sinus surgery on lung function, IV antibiotic use, and hospitalization in 15 adults with CF. 12 month pre-operative and 12 month post-operative forced vital capacity (FVC) and FEV1, IV antibiotic courses, total number of days IV use, number of inpatient hospital days (IHD) were assessed. They found a significant reduction in the number of IHDs in the postoperative period ( 36.7 days vs $59.1, \mathrm{p}=0.03$ ) without evidence of improved lung function or the need for IV antibiotics. Similarly, in a study examining the effect of endoscopic sinus surgery on the pulmonary status of adults with 
cystic fibrosis [20], 12 month pre and post-operative FEV1, days hospitalized, and days of IV antibiotics was assessed in 32 patients. The authors found that endoscopic sinus surgery did not reduce days hospitalized or days on IV for a respiratory exacerbation in the pre- vs postoperative period and concluded that there was no effect of FESS on progression of lung disease in patients with CF.

A recent study by Alanin et al [21] examined chronic Gram-negative lung infection 3 years post-endoscopic sinus surgery in 106 patients with cystic fibrosis including pediatric and lung transplant patients. Similar to our findings, the authors found that lung function declined in the 3-year post-operative period. Although the number of gram-negative bacteria non-colonized and intermittently colonized patients significantly increased over the study period (16/106 patients pre-operatively (15\%) vs 35/106 patients (33\%) post-operatively and $61(58 \%)$ to $34(32 \%)$ respectively) after three years, the number of chronically infected patients increased from $29(27 \%)$ to $37(35 \%)$. They also found that SNOT-22 scores improved while the global quality of life scores decreased.

More recently, Khalfoun et al [22] performed a retrospective chart review of 181 patients who underwent FESS from January 2009 to July 2014. Lung function data, including the forced expiratory volume in one second (FEV1), forced vital capacity (FVC), and FEV1/FVC, were retrieved within 1 year before and after surgery. They found that for the entire cohort, lung function did not change related to FESS. Among patients with FEV1 <80\%, FEV1 declined presurgery by $3.5 \%$ per year $(95 \%$ CI, $-6.1 \%$ to $-0.8 \%$; P = .010), which halted after surgery with these patients, then showing no subsequent change in FEV1 (95\% CI, 0.9\%-3.7\%; $\mathrm{P}=.240$ ). No benefit was identified for patients with FEV1 $>80 \%$. They concluded that their findings suggest FESS may benefit pulmonary outcomes.

\section{Clinical Applicability of the Study}

Overall, most studies do not show a significant benefit of ESS on pulmonary outcomes in CF patients. This may be due to a lack of consensus regarding type of surgery offered (full ESS vs uncinectomy/maxillary antrostomy), immediate post-operative sinonasal regime (i.e. duration of IV/oral antibiotic therapy and topical management), appropriate patient selection (mutation type, lung transplantation status, etc.) and frequency of long-term follow-up with management of sinonasal exacerbations. Interestingly, in our study, we identified an increase in the total number of days hospitalized post-operatively. This may be due to the fact that we are performing surgical intervention on patients with more severe disease and the natural course of their disease leads to an increased total number of days hospitalized.

\section{Strengths of the Study}

Our study is one of the largest studies examining the effect ESS on pulmonary outcomes in the CF population and the only study to examine outcomes 2 year post-operatively. This study was limited by its retrospective nature and small cohort size, which is inherent in the CF population. Symptom and endoscopic scores were not examined. The findings of this study can guide further management and research examining sinonasal disease and sinus surgery in the CF population.

Similar to current literature, our results did not show a significant benefit of endoscopic sinus surgery on clinical outcomes including FEV1 and pulmonary exacerbations. Further studies are required to determine improvement in quality of life in patients undergoing endoscopic sinus surgery. Studies examining the effect of aggressive follow-up and management of sinonasal disease in this population are warranted.

\section{CONFLICT OF INTEREST}

This study received no funding. There is no potential or actual conflict of interest with respect to this study.

\section{DATA SHARING}

The data that support the findings of this study are available from the corresponding author upon reasonable request.

\section{FUNDING STATEMENT}


This study received no funding.

\section{REFERENCES}

1. Rowe SM, Miller S, Sorscher EJ (2005) Cystic fibrosis. N Engl J Med 352(19):1992-2001.

2. Canadian Cystic Fibrosis Registry (2013) Toronto, Ontario.

3. Riordan JR RJ, Kerem B et al (1989) Identification of the cystic fibrosis gene: cloning and characterization of complementary DNA. Science 245(4922):1066-73.

4. Sheppard D WM (1999) Structure and function of the CFTR chloride channel. Physiol Rev 79:S23-S45.

5. Regnis JA RM, Bailey DL et al (1994) Mucociliary clearance in patients with cystic fibrosis and in normal subjects. Am J Respir Crit Care Med 150:66-71.

6. Bethesda M (2011) Annual Data Report. Cystic Fibrosis Foundation Patient Registry.

7. Cimmino M, Cavaliere M, Nardone M, Plantulli A, Orefice A, Esposito V et al (2003) Clinical characteristics and genotype analysis of patients with cystic fibrosis and nasal polyposis. Clin Otolaryngol Allied Sci 28(2):125-32.

8. Sakano E, Ribeiro AF, Barth L, Condino Neto A, Ribeiro JD (2007) Nasal and paranasal sinus endoscopy, computed tomography and microbiology of upper airways and the correlations with genotype and severity of cystic fibrosis. Int J Pediatr Otorhinolaryngol 71(1):41-50.

9. Yung MW, Gould J, Upton GJ. (2002) Nasal polyposis in children with cystic fibrosis: a long-term follow-up study. Ann Otol Rhinol Laryngol 111(12 Pt 1):1081-6.

10. Aanaes K, von Buchwald C, Hjuler T, Skov M, Alanin M, Johansen HK (2013) The effect of sinus surgery with intensive follow-up on pathogenic sinus bacteria in patients with cystic fibrosis. Am J Rhinol Allergy 27(1):e1-4.

11. Johansen H AK, Pressler T et al (2012) Colonisation and infection of the paranasal sinuses in cystic fibrosis patients is accompanied by a reduced PMN response. J Cyst Fibros 11:525-31.

12. Munck A BS, Mariani-Kukdijan P et al (2001) Genotypic characterization of Pseudomonas aeruginosa strains recovered from patients with cystic fibrosis after initial and subsequent colonization. Pediatr Pulmonol $32: 288-92$.

13. Dosanijh A LS, Elashoff D et al (2000) A comparison of microbiologic flora of the sinuses and airway among cystic fibrosis patients with maxillary antrostomies. Pediatr Transplant 4(3):182-5.

14. Chang EH (2014) New insights into the pathogenesis of cystic fibrosis sinusitis. Int Forum Allergy Rhinol $4(2): 132-7$.

15. Desrosiers M, Evans GA, Keith PK, Wright ED, Kaplan A et al (2011) Canadian clinical practice guidelines for acute and chronic rhinosinusitis. J Otolaryngol Head Neck Surg 40 (Suppl 2):S99-193.

16. Shatz A (2006) Management of recurrent sinus disease in children with cystic fibrosis: a combined approach. Otolaryngol Head Neck Surg 135(2):248-52.

17. Liang J, Higgins TS, Ishman SL, Boss EF, Benke JR, Lin SY (2013) Surgical management of chronic rhinosinusitis in cystic fibrosis: a systematic review. Int Forum Allergy Rhinol 3(10):814-22.

18. Macdonald KI1, Gipsman A, Magit A, Fandino M, Massoud E, Witterick IJ, Hong P (2012) Endoscopic sinus surgery in patients with cystic fibrosis: a systematic review and meta-analysis of pulmonary function. Rhinology 50(4):360-9. 
19. Henriquez OA, Wolfenden LL, Stecenko A, Delgaudio JM, Wise SK (2012) Endoscopic sinus surgery in adults with cystic fibrosis: effect on lung function, intravenous antibiotic use, and hospitalization. Arch Otolaryngol Head Neck Surg 138(12):1167-70.

20. Kempainen RR, Sajan JA, Pylkas AM, Dunitz JM, Rimell FL, Milla CE (2012) Effect of endoscopic sinus surgery on pulmonary status of adults with cystic fibrosis. Otolaryngol Head Neck Surg 147(3):557-62.

21. Alanin MC, Aanaes K, Høiby N, Pressler T, Skov M, Nielsen KG, Taylor-Robinson D, Waldmann E, Krogh Johansen H, von Buchwald C (2016) Sinus surgery postpones chronic Gram-negative lung infection: cohort study of 106 patients with cystic fibrosis. Rhinology 54(3):206-13.

22. Khalfoun S, Tumin D, Ghossein M, Lind M, Hayes D Jr, Kirkby S (2018) Improved lung function after ainus surgery in cystic fibrosis patients with moderate obstruction. Otolaryngol Head Neck Surg 158(2):381385.

\section{Hosted file}

Table 1.docx available at https://authorea.com/users/322781/articles/451705-effect-ofendoscopic-sinus-surgery-on-clinical-outcomes-in-cystic-fibrosis

\section{Hosted file}

Table 2.docx available at https://authorea.com/users/322781/articles/451705-effect-ofendoscopic-sinus-surgery-on-clinical-outcomes-in-cystic-fibrosis

\section{Hosted file}

Table 3.docx available at https://authorea.com/users/322781/articles/451705-effect-ofendoscopic-sinus-surgery-on-clinical-outcomes-in-cystic-fibrosis

\section{Hosted file}

Table 4.docx available at https://authorea.com/users/322781/articles/451705-effect-ofendoscopic-sinus-surgery-on-clinical-outcomes-in-cystic-fibrosis

\section{Hosted file}

Table 5.docx available at https://authorea.com/users/322781/articles/451705-effect-ofendoscopic-sinus-surgery-on-clinical-outcomes-in-cystic-fibrosis 